\title{
The Design of Fixed-Time Observer and Finite-Time Fault-Tolerant Control for Hypersonic Gliding Vehicles
}

\author{
Xiang Yu, Senior Member, IEEE, Peng Li, and Youmin Zhang, Senior Member, IEEE
}

\begin{abstract}
This paper proposes a fault-tolerant control scheme for a hypersonic gliding vehicle to counteract actuator faults and model uncertainties. Starting from the kinematic and aerodynamic models of the hypersonic vehicle, the control-oriented model subject to actuator faults is built. The observers are designed to estimate the information of actuator faults and model uncertainties, and to guarantee the estimation errors for converging to zero in fixed settling time. Subsequently, the finite-time multivariable terminal sliding mode control and composite-loop design are pursued to enable integration into the faulttolerant control, which can ensure the safety of the postfault vehicle in a timely manner. Simulation studies of a six degree-of-freedom nonlinear model of the hypersonic gliding vehicle are carried out to manifest the effectiveness of the investigated fault-tolerant control system.
\end{abstract}

Index Terms-Hypersonic gliding vehicle, actuator faults and model uncertainties, fixed-time observer, finite-time fault-tolerant control.

\section{INTRODUCTION}

A Hypersonic gliding vehicle (HGV) is launched into the sub-orbital trajectory either by a booster rocket or a reusable launch vehicle. As a result, the HGV can maneuver with sub-orbital velocities and in the near space (from 20 $\mathrm{km}$ to $100 \mathrm{~km}$ in altitude). HGVs are intended to be an excellent technology, due to their high degrees of long-range delivery capability and maneuvering flexibility. Back-stepping [2], robust control [3], adaptive control [4], model predictive control (MPC) [5], sliding mode control (SMC) [6], and feedback linearization [7] approaches have been proposed for improving the control performance and eliminating the influence of model uncertainties.

This work was supported in part by NSERC, in part by the National Natural Science Foundation of China under Grants 61403407 , 61573282 , and 61603130, in part by the Natural Science Foundation of Shaanxi Province under Grant 2015JZ020, and in part by the Research Fund of State Key Laboratory of High Performance Computing (NUDT) under Grant 201613-02.

Xiang Yu (xiangyu1110@gmail.com) and Youmin Zhang (Corresponding Author; youmin.zhang@concordia.ca) are with the Department of Mechanical, Industrial and Aerospace Engineering, Concordia University, Montreal, Quebec, H3G 1M8, Canada.

Peng Li (lipeng_2010@163.com) is with the College of Mechatronics Engineering and Automation, and the State Key Laboratory of High Performance Computing, National University of Defense Technology, Changsha, 410073, China.
The increasing complexity and automation render the HGVs susceptible to component/system faults. Substantial performance degradation and even catastrophic consequences can be attributed to in-flight failures. Over the past decades, the growing demand for safety, reliability, maintainability, and survivability in safety-critical systems has motivated significant research in fault detection and diagnosis (FDD) [8][10] and fault-tolerant control (FTC) [11]-[18]. FTC can accommodate faults among system components automatically and maintain system safety with an acceptable degree of overall performance.

The FTC design with application to hypersonic vehicles is the result of several years of research. Adaptive FTC laws are presented to mitigate the adverse effects due to partial loss of actuator effectiveness [19] and time-varying actuator faults [20], respectively. By resorting to MPC and reference reshaping techniques, an FTC system is proposed to cancel the effect of actuator partial failures [21]. It is also noteworthy that the SMC techniques are exploited at the FTC design stage of hypersonic vehicles [22], [24]-[26]. Sliding mode based FTC is deployed for attitude control of a hypersonic vehicle, ensuring the asymptotic tracking under actuator faults [22]. The authors develop an adaptive FTC scheme against multiple faults of a hypersonic vehicle [23]. As can be summarized in [24], [25], the FTC design is presented on the basis of backstepping and SMC methods, counteracting actuator faults. An FTC scheme, which is composed by an SMC and a nonlinear disturbance observer, is applied to a hypersonic aircraft with the limited control authority [26].

Although various degrees of success in HGV FTC design have been achieved, there still exist several problems to be further investigated. 1) From a safety point of view, it is highly desirable that the fault diagnosis and the corresponding accommodation can be completed in a timely manner [15], [16]. The pivotal early works in [27], [28] discuss finite-time stability of nominal systems. In consequence, several results are available in the literature that consider finite-time control techniques with fast convergence rate and high precision control performance [29]-[33]. Furthermore, the concept of fixed-time stability independent of system initial conditions is preliminarily discovered [34]-[38]. To the best of the authors' knowledge, how to adopt finite/fixed-time stability in the HGV fault diagnosis and then accommodation requires extensive research. 2) Due to short of wind tunnel facilities and flight experiments, a severe difficulty in designing $\mathrm{HGV}$ control 
systems results from the large uncertainties and perturbations inherent to the HGV model [1]. Not only uncertainty exists in dynamic matrix, but also in control input matrix. This factor renders a great challenge of FTC design. Furthermore, the existing design is enforced to inner-loop control and outerloop control based on the kinematic model of attitude (bank angle, angle of attack, and sideslip angle) and the dynamic model of attitude (roll rate, pitch rate, and yaw rate). The so-called separate design is relatively simple according to the timescale separation principle [39]. Nonetheless, the stability of the overall system cannot be guaranteed from a theoretical perspective. 3) Sliding mode observer (SMO) and SMC start being exploited for HGV FTC system design. Nevertheless, in most of the SMO and SMC approaches proposed to date, a multi-input problem with $m$ inputs is recast as a decoupled problem involving $m$ single-input structures. Multivariable design of SMO and SMC is demanded rather than the decoupled treatment, by considering strong couplings and nonlinearity inherent to an HGV.

Motivated by the aforementioned difficulties, particular attention is devoted to achieving fixed-time fault estimation and finite-time FTC within the context of multivariable design. Thus, the developed FTC scheme can provide accommodation for HGV actuator faults and model uncertainties. The major contributions are stated as follows.

1) When comparing to the finite-time SMO [40]-[42] and the sliding mode based disturbance observer [43]-[46], the proposed fixed-time SMO can ensure that the estimation error of the "lumped disturbance" converges to a small vicinity of zero in fixed time. Moreover, the convergence time of the designed disturbance observer is independent of initial conditions, while elegant solutions can be achieved by vector form design.

2) The FTC presented in this paper is integrated by fixedtime SMO and finite-time control. The resulting FTC can promptly enforce the trajectory of the faulty HGV converging to a small vicinity of origin, without the need of excessive control efforts. It should be mentioned that both model uncertainties and actuator faults are explicitly considered over the design phase. By resorting to the proposed FTC scheme, corrective reactions can be taken in response to the actuator faults for fulfilling the stringent requirement of HGV safety. To the best of the authors' knowledge, there exist few papers focusing on this aspect.

3) In opposition to the multivariable SMC design for a hypersonic vehicle in [40], the developed approach of this study can avoid the problem associated with the decoupled design and ensure the globally finite-time stability in spite of actuator faults. This study is applicable especially in the case where the strong couplings are exposed on HGV aerodynamics. The use of multivariable design avoids the necessity for the decoupled design with $m$ single-input and single-output (SISO) structures. These improvements have the potential of enhancing the safety of operational HGVs, since the coupling and inherent functional redundancy of an $\mathrm{HGV}$ have been better exploited in such a multivariable design approach.

The rest of this paper is arranged as follows. The controloriented HGV model, actuator fault mode, and problem statement are given in Section II. An HGV FTC scheme is proposed against actuator malfunctions and model uncertainties in Section III, where the fixed-time SMO and the finite-time SMC based FTC are presented with the aid of multivariable design. In Section IV, the performance of the developed FTC is evaluated by means of simulations of a full nonlinear HGV model. Section V includes a discussion of the conclusions.

\section{HGV Modeling And Problem Statement}

\section{A. HGV Dynamics}

The HGV is modeled based on the assumption of a rigid vehicle structure, a flat, non-rotating Earth and uniform gravitational field. Therefore, the inertial position coordinates are written as:

$$
\left\{\begin{array}{l}
\dot{x}=V \cos \gamma \cos \chi \\
\dot{y}=V \cos \gamma \sin \chi \\
\dot{z}=-V \sin \gamma
\end{array}\right.
$$

where $x, y$, and $z$ stand for the positions with respect to $x, y$, and $z$ directions of the Earth-fixed reference frame, $V$ specifies the total velocity of the HGV, $\chi$ and $\mu$ represent the flight-path angle and the bank angle, respectively.

The force equations are expressed as:

$$
\left\{\begin{array}{l}
\dot{V}=-g \sin \gamma-\frac{Q S_{r} C_{D}}{m} \\
\dot{\chi}=\frac{Q S_{r}}{m V \cos \gamma}\left(C_{L} \sin \mu+C_{Y} \cos \mu\right) \\
\dot{\gamma}=-\frac{g}{V} \cos \gamma+\frac{Q S_{r}}{m V}\left(C_{L} \cos \mu-C_{Y} \sin \mu\right)
\end{array},\right.
$$

where $g, Q, S_{r}, m$, and $\gamma$ denote the gravitational constant, the dynamic pressure, the reference area, the mass of the $\mathrm{HGV}$, and the heading angle, $C_{L}, C_{D}$, and $C_{Y}$ are the aerodynamic coefficients with respect to lift, drag, and side force, respectively.

The kinematic model of attitude is described as:

$$
\left\{\begin{aligned}
\dot{\mu}= & \sec \beta(p \cos \alpha+r \sin \alpha) \\
& +\frac{Q S_{r} C_{L}}{m V}(\tan \gamma \sin \mu+\tan \beta) \\
& +\frac{Q S_{r} C_{Y}}{m V} \tan \gamma \cos \mu-\frac{g}{V} \cos \gamma \cos \mu \tan \beta \\
\alpha & =q-\tan \beta(p \cos \alpha+r \sin \alpha) \\
& +\frac{1}{m V \cos \beta}\left(m g \cos \gamma \cos \mu-Q S_{r} C_{L}\right) \\
\dot{\beta}= & -r \cos \alpha+p \sin \alpha \\
& +\frac{1}{m V}\left(Q S_{r} C_{Y}+m g \cos \gamma \sin \mu\right)
\end{aligned}\right.
$$

where $\alpha$ and $\beta$ denote the angle of attack (AOA) and the sideslip angle, respectively.

The dynamic model of attitude is given as:

$$
\left\{\begin{array}{l}
\dot{p}=\frac{Q S_{r} \bar{b} C_{l}}{I_{x x}} \\
\dot{q}=\frac{Q S_{r} b C_{m}+\left(I_{z z}-I_{x x}\right) p r}{I_{y y}} \\
\dot{r}=\frac{Q S_{r} \bar{b} C_{n}+\left(I_{x x}-I_{y y}\right) p q}{I_{z z}}
\end{array},\right.
$$

where $\bar{b}$ denotes the wing span of the $\mathrm{HGV}, C_{l}, C_{m}$, and $C_{n}$ represent the moment coefficients of the rolling, pitching, and yawing channels, $I_{x x}, I_{y y}$, and $I_{z z}$ denote moments of inertia with respect to $x, y$, and $z$ coordinate, respectively. 
The aerodynamic force and moment coefficients are:

$$
\begin{aligned}
& C_{D}=C_{D}(M, \alpha) \\
& C_{Y}=C_{Y, \beta}(M, \alpha) \beta+C_{Y, \delta_{r}}(M, \alpha) \delta_{r} \\
& C_{L}=C_{L}(M, \alpha)+C_{L, \delta_{e}}(M, \alpha) \delta_{e} \\
& C_{l}=C_{l, \beta}(M, \alpha) \beta+C_{l, \delta_{a}}(M, \alpha) \delta_{a}+C_{l, p}(M, \alpha) \frac{p \bar{b}}{2 V} \\
& C_{m}=C_{m, \alpha}(M, \alpha)+C_{m, \delta_{e}}(M, \alpha) \delta_{e}+C_{m, q}(M, \alpha) \frac{q \bar{b}}{2 V} \\
& C_{n}=C_{n, \beta}(M, \alpha) \beta+C_{n, \delta_{r}}(M, \alpha) \delta_{r}+C_{n, r}(M, \alpha) \frac{r b}{2 V}
\end{aligned}
$$

where $\delta_{a}, \delta_{e}$, and $\delta_{r}$ denote the control deflections of the aileron, elevator, and rudder, respectively. Note that $C_{Y, \beta}$ and $C_{Y, \delta_{r}}$ are the partial derivatives of $C_{Y}$ with respect to $\beta$ and $\delta_{r}$, respectively. $C_{L, \delta_{e}}$ stands for the partial derivative of $C_{L}$ with respect to $\delta_{e} . C_{l, \beta}, C_{l, \delta_{a}}$, and $C_{l, p}$ are the partial derivatives of $C_{l}$ with respect to $\beta, \delta_{a}$, and $p$, respectively. $C_{m, \alpha}, C_{m, \delta_{e}}$, and $C_{m, q}$ are the partial derivatives of $C_{m}$ with respect to $\alpha$, $\delta_{e}$, and $q$, respectively. $C_{n, \beta}, C_{n, \delta_{r}}$, and $C_{n, r}$ are the partial derivatives of $C_{n}$ with respect to $\beta, \delta_{r}$, and $r$, respectively.

\section{B. Control-Oriented Model Subject to Actuator Faults}

The control-oriented model of the $\mathrm{HGV}$ is established by combining the kinematic model and the dynamic model of the HGV attitude, based on which the so-called composite-loop FTC design can be achieved.

As far as an attitude control system is concerned, $\mu, \alpha$, and $\beta$ can be gathered into a vector $x_{1}=[\mu, \alpha, \beta]^{T}$. In terms of Eq. (3), one can obtain:

$$
\left\{\begin{array}{l}
\dot{\mu}=\sec \beta(p \cos \alpha+r \sin \alpha)+f_{\mu} \\
\dot{\alpha}=q-\tan \beta(p \cos \alpha+r \sin \alpha)+f_{\alpha} \\
\dot{\beta}=-r \cos \alpha+p \sin \alpha+f_{\beta}
\end{array}\right.
$$

where

$$
\left\{\begin{array}{rl}
f_{\mu}= & \frac{Q S C_{L}}{m V}(\tan \gamma \sin \mu+\tan \beta) \\
& +\frac{Q S C_{Y}}{m V} \tan \gamma \cos \mu-\frac{g}{V} \cos \gamma \cos \mu \tan \beta \\
f_{\alpha}= & \frac{m_{1}}{m V \cos \beta}\left(m g \cos \gamma \cos \mu-Q S C_{L}\right) \\
f_{\beta}= & \frac{1}{m V}\left(Q S C_{Y} \cos \beta+m g \cos \gamma \sin \mu\right)
\end{array} .\right.
$$
as:

By defining $x_{2}=[p, q, r]^{T}$, Eqs. (6)-(7) can be described

$$
\dot{x}_{1}=f_{1}+g_{1} x_{2},
$$

where $f_{1}=\left[f_{\mu}, f_{\alpha}, f_{\beta}\right]^{T}$ and

$$
g_{1}=\left[\begin{array}{ccc}
\sec \beta \cos \alpha & 0 & \sec \beta \sin \alpha \\
-\tan \beta \cos \alpha & 1 & -\tan \beta \sin \alpha \\
\sin \alpha & 0 & -\cos \alpha
\end{array}\right] .
$$

By accounting for Eqs. (4)-(5), one can render:

$$
\left\{\begin{array}{l}
\dot{p}=f_{p}+\frac{Q S_{r} \bar{b} C_{l, \delta_{a}}}{I_{x x}} \delta_{a} \\
\dot{q}=f_{q}+\frac{Q S_{r} \bar{b} C_{m, \delta_{e}}}{I_{y y}} \delta_{e} \\
\dot{r}=f_{r}+\frac{Q S_{r} \bar{b} C_{n, \delta_{r}}}{I_{z z}} \delta_{r}
\end{array}\right.
$$

where

$$
\left\{\begin{array}{rl}
f_{p} & =\frac{Q S_{r} \bar{b}\left(C_{l, \beta} \beta+C_{l, p} \frac{p \bar{b}}{2 V}\right)}{I_{x x}} \\
f_{q} & =\frac{Q S_{r} \bar{b}\left(C_{m, \alpha}+C_{m, q} \frac{q \bar{b}}{2 V}\right)+\left(I_{z z}-I_{x x}\right) p r}{I_{y y}} \\
f_{r} & =\frac{Q S_{r} \bar{b}\left(C_{n, \beta} \beta+C_{n, r} \frac{r \bar{b}}{2 V}\right)+\left(I_{x x}-I_{y y}\right) p q}{I_{z z}}
\end{array} .\right.
$$

Further, Eqs. (10)-(11) can be formed as:

$$
\dot{x}_{2}=f_{2}+g_{2} u,
$$

where $f_{2}=\left[f_{p}, f_{q}, f_{r}\right]^{T}, u=\left[\delta_{a}, \delta_{e}, \delta_{r}\right]^{T}$, and

$$
g_{2}=\left[\begin{array}{ccc}
\frac{Q S_{r} \bar{b} C_{l, \delta_{a}}}{I_{x x}} & 0 & 0 \\
0 & \frac{Q S_{r} \bar{b} C_{m, \delta_{e}}}{I_{y y}} & 0 \\
0 & 0 & \frac{Q S_{r} \bar{b} C_{n, \delta_{r}}}{I_{z z}}
\end{array}\right] .
$$

Gain fault and bias fault are the faults commonly occurring on flight actuators. In this work, the actuator fault model including both sorts of faults is generally formed as:

$$
u_{F}=\Lambda u+\rho,
$$

where $\Lambda=\operatorname{diag}\left\{\lambda_{1}, \lambda_{2}, \lambda_{3}\right\}$ represents the gain fault and $\rho=$ $\left[\rho_{a}, \rho_{e}, \rho_{r}\right]^{T}$ denotes the bias fault, respectively. Note that $0<$ $\lambda_{i} \leq 1, i=1,2,3$. Thus, in the presence of actuator faults, Eq. (12) is represented as:

$$
\begin{aligned}
\dot{x}_{2} & =f_{2}+g_{2}(\Lambda u+\rho) \\
& =f_{2}+g_{2} u+g_{2}(\Lambda-I) u+g_{2} \rho
\end{aligned} .
$$

Note that $u$ in Eqs. (12), (14), and (15) represents the control input vector under normal conditions, while $u_{F}$ describes the control input vector in the case of faults.

Assumption 1: It is assumed that the condition $\left\|g_{2}(\Lambda-I) g_{2}^{-1}\right\|_{\infty}<1$ holds.

Remark 1: There exists a condition $\left\|g_{2}(\Lambda-I) g_{2}^{-1}\right\|_{\infty}<$ 1 , such that the control signals $g_{2} u$ dominate the fault vector function $g_{2}(\Lambda-I) u$ [47].

By accounting for the actuator faults and model uncertainties, the control-oriented model in vector format is established as:

$$
\left\{\begin{array}{l}
\dot{x}_{1}=g_{1} x_{2}+\Delta_{1} \\
\dot{x}_{2}=f_{2}+g_{2} u+\Delta_{2}
\end{array},\right.
$$

where $\Delta_{1}$ arises from $f_{1}$, and $\Delta_{2}=g_{2}(\Lambda-I) u+g_{2} \rho$ is the lumped uncertainty induced by actuator faults.

By defining $y_{1}=x_{1}-x_{1, d}$ and $y_{2}=g_{1} x_{2}-\dot{x}_{1, d}$, the following equations can be achieved:

$$
\left\{\begin{array}{l}
\dot{y}_{1}=y_{2}+\Delta_{1} \\
\dot{y}_{2}=\dot{g}_{1} x_{2}+g_{1} f_{2}-\ddot{x}_{1, d}+g_{1} g_{2} u+g_{1} \Delta_{2}
\end{array},\right.
$$

where $x_{1, d}$ represents the desired states. Letting $a(\cdot)=\dot{g}_{1} x_{2}+$ $g_{2} f_{2}-\ddot{x}_{1, d}, b(\cdot)=g_{1} g_{2}$, and $\Delta_{3}=g_{1} \Delta_{2}$, Eq. (17) can be simplified as:

$$
\left\{\begin{array}{l}
\dot{y}_{1}=y_{2}+\Delta_{1} \\
\dot{y}_{2}=a+b u+\Delta_{3}
\end{array} .\right.
$$

Remark 2: As can be seen from Fig. 1 and also Eq. (8), the input vector of the outer-loop HGV model consists of the roll rate $(p)$, pitch rate $(q)$, and yaw rate $(r)$, while the state vector with respect to the outer-loop is composed by the bank angle $(\mu)$, AOA $(\alpha)$, and sideslip angle $(\beta)$, respectively. Focusing on the inner-loop HGV model of Eq. (12), the deflections of the aileron $\left(\delta_{a}\right)$, elevator $\left(\delta_{e}\right)$, and rudder $\left(\delta_{r}\right)$ are regarded as the inputs, while the roll rate, pitch rate, and yaw rate constitute the state vector. It should be mentioned that $f_{2}$ in Eq. (12) is closely related to the states of Eq. (8).

Remark 3: Unpredictable aerodynamics due to hypersonic speed and airframe/structural dynamics interactions constitute the uncertainty source. As can be seen from Eq. (7), the aerodynamic coefficients $C_{L}$ and $C_{Y}$ with uncertainty are 
contained in $f_{1}$. Hence, $f_{1}=\Delta_{1}$ in Eq. (16) is regarded as the model uncertainty. Moreover, the term in Eq. (18), $\Delta_{3}=g_{1} g_{2}(\Lambda-I) u+g_{1} g_{2} \rho$, includes the information of actuator faults, without a priori knowledge. In the following, fixed-time observers are developed to estimate $\Delta_{1}$ and $\Delta_{3}$, respectively.

Remark 4: Note that $\Delta_{1}=f_{1}=\left[f_{\mu}, f_{\alpha}, f_{\beta}\right]^{T}$. In Eq. (6), $f_{\mu}, f_{\alpha}$, and $f_{\beta}$ are regarded as the impact terms of trajectory on the HGV attitude. The value of $V$ is usually very large over HGV flight envelopes. Furthermore, $\beta \approx 0$ and $\gamma \neq \pm \pi / 2$. Therefore, the assumption that $\Delta_{1}$ is bounded is reasonable. Focusing on $\Delta_{3}=g_{1} g_{2}(\Lambda-I) u+g_{1} g_{2} \rho, \Delta_{3}$ is related to system states and control inputs, which are bounded. With respect to $\mathrm{HGV}$ fight envelopes, $\beta \approx 0$ and each element of $g_{2}$ is composed by bounded control moment coefficients. In consequence, $\Delta_{3}$ is bounded in flight.

Remark 5: As reported in [48], hydraulic driven actuators are configured in hypersonic vehicles to operate all control surfaces. Flush air data system (FADS) that is often mounted in the upper and lower lifting surfaces has been successfully applied to hypersonic vehicles [49]. FADS, which is dependent on the pressure sensor array measurement of aircraft surface pressure distribution, obtains dynamic pressure, bank angle, AOA, and sideslip angle indirectly through a specific algorithm. In addition, an inertial navigation system (INS) can measure the position, orientation, and velocity of a hypersonic vehicle. Hence, with respect to the studied HGV, the bank angle, AOA, and sideslip angle can be measured by an FADS, while the measurements of the angular rates of roll, pitch, and yaw can be provided by an INS.

Remark 6: It is reported that in [50] that the leakage of hydraulic fluid can be the root cause of the degradation of the actuator effectiveness. Therefore, $\Lambda=\operatorname{diag}\left\{\lambda_{1}, \lambda_{2}, \lambda_{3}\right\}$ in Eq. (14) is used to describe the effectiveness of the HGV actuators, where $0<\lambda_{1}, \lambda_{2}, \lambda_{3} \leq 1$. In addition, an actuation system in aircraft is constructed by an actuator controller, an actuator, and sensors. The sensor fault in an actuator system is recognized as the one of the major causes of the actuator bias faults. If the amplitude sensor encounters a bias fault, the measured amplitude is the actual amplitude plus the bias value. As a consequence, the sensed amplitude is controlled to be equal to the referenced signal. However, the actual value of the actuator amplitude is deviated from the one as required by the flight control. Hence, $\rho=\left[\rho_{a}, \rho_{e}, \rho_{r}\right]^{T}$ is adopted in Eq. (14) to describe the bias faults of the aileron, elevator, and rudder, respectively.

\section{Problem Statement}

The objective is to design an FTC scheme such that:

1) The terms including actuator faults and system uncertainties can be estimated within a fixed amount of time, thus:

$$
\lim _{t \rightarrow t_{o}}\left\|\hat{\Delta}_{1}-\Delta_{1}\right\|=0, \lim _{t \rightarrow t_{o}}\left\|\hat{\Delta}_{3}-\Delta_{3}\right\|=0
$$

where $t_{o}$ is the fixed convergence time, $\hat{\Delta}_{1}$ and $\hat{\Delta}_{3}$ are the estimates of $\Delta_{1}$ and $\Delta_{3}$, respectively.

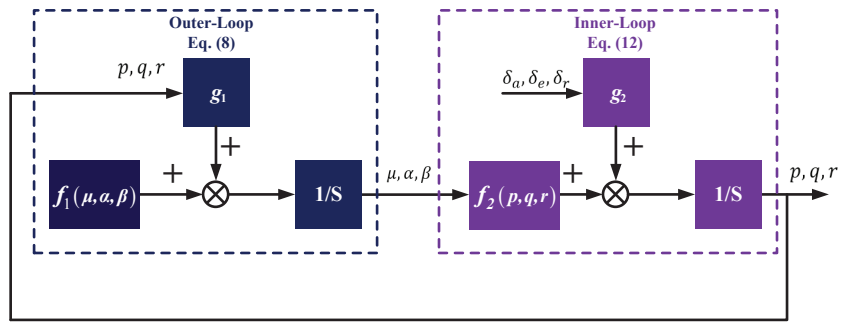

Fig. 1. Block diagram of the HGV model.

2) The detrimental impact of HGV actuator faults can be counteracted within a finite amount of time, thus:

$$
\lim _{t \rightarrow t_{c}}\left|\mu-\mu_{d}\right|=0, \lim _{t \rightarrow t_{c}}\left|\alpha-\alpha_{d}\right|=0, \lim _{t \rightarrow t_{c}}\left|\beta-\beta_{d}\right|=0
$$

where $t_{c}$ denotes the finite convergence time, $\mu_{d}, \alpha_{d}$, and $\beta_{d}$ correspond to the reference signals of the bank angle, AOA, and sideslip angle, respectively.

3) The composite-loop design is achieved under multivariable situation, by which separating the HGV dynamics into inner and outer loops is no longer needed.

\section{Fixed-Time Observer Design}

\section{A. An Overview of the Developed FTC Architecture}

As illustrated in Fig. 2, the developed FTC scheme consists of a fixed-time observer and a finite-time FTC unit. Once the HGV encounters actuator faults, the observer can be continuously run in an effort to obtaining the information within fixed settling time. Then, the FTC responds to the observer results, ensuring the states of the handicapped HGV approach to the desired ones within finite time. Therefore, two problems are addressed in the following. The first is the observer design by means of the fixed-time multivariable sliding mode technique. The second is the synthesis of the FTC against actuator faults and model uncertainties, using the finite-time multivariable integral terminal SMC (TSMC) method.

Remark 7: It is of paramount importance to investigate sensor faults in engineering plants as well [51]-[53]. When a sensor is lost due to malfunctions, reliable information can no longer be sent to the flight control system and the flight management system, having a deleterious impact on vehicle's safety. In terms of sensor FTC, appropriate reconstructions can be applied to replace the sensor measurements before the erroneous information is delivered to the controller. Nonetheless, more emphasis is placed against actuator faults in this study due to the limited space.

\section{B. Fixed-Time Observer}

Theorem 1: Consider the faulty system described by Eq. (18), and assume that the terms $\Delta_{1}$ and $\Delta_{3}$ satisfy $\left\|\dot{\Delta}_{1}\right\| \leq$ $L_{1}$ and $\left\|\dot{\Delta}_{3}\right\| \leq L_{2}$, where $L_{1}$ and $L_{2}$ are known constants. Define $z_{1}, z_{2}, z_{3}$, and $z_{4}$ as the states of the designed fixedtime observers. If the observers are designed by Eqs. (21)-(22) 


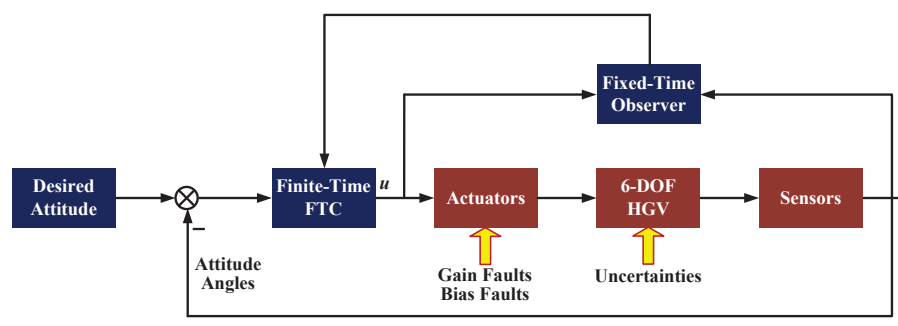

Fig. 2. Conceptual HGV FTC block diagram.

under the condition (23), therefore the terms $\Delta_{1}$ and $\Delta_{3}$ can be estimated within fixed time through $z_{2}$ and $z_{4}$, respectively.

$$
\begin{gathered}
\left\{\begin{array}{l}
\dot{z}_{1}=-l_{1} \frac{e_{1}}{\left\|e_{1}\right\|^{1 / 2}}-l_{2} e_{1}\left\|e_{1}\right\|^{p-1}+z_{2}+y_{2}, \\
\dot{z}_{2}=-l_{3} \frac{e_{1}}{\left\|e_{1}\right\|}
\end{array}\right. \\
\left\{\begin{array}{l}
\dot{z}_{3}=-l_{4} \frac{e_{2}}{\left\|e_{2}\right\|^{1 / 2}}-l_{5} e_{2}\left\|e_{2}\right\|^{p-1}+z_{4}+a+b u \\
\dot{z}_{4}=-l_{6} \frac{e_{2}}{\left\|e_{2}\right\|}
\end{array}\right.
\end{gathered}
$$

where $e_{1}=z_{1}-y_{1}, e_{2}=z_{3}-y_{2}, p>1$, and

$$
\left\{\begin{array}{l}
l_{1}>\sqrt{2 l_{3}}, l_{2}>0, l_{3}>4 L_{1} \\
l_{4}>\sqrt{2 l_{6}}, l_{5}>0, l_{6}>4 L_{2}
\end{array} .\right.
$$

Proof. By taking the derivative of $e_{1}$, one can obtain that:

$$
\begin{aligned}
\dot{e}_{1} & =\dot{z}_{1}-\dot{x}_{1} \\
& =-l_{1} \frac{e_{1}}{\left\|e_{1}\right\|^{1 / 2}}-l_{2} e_{1}\left\|e_{1}\right\|^{p-1}+z_{2}-\Delta_{1} .
\end{aligned}
$$

Letting $e_{1}^{*}=z_{2}-\Delta_{1}$, Eq. (24) can be rewritten as:

$$
\dot{e}_{1}=-l_{1} \frac{e_{1}}{\left\|e_{1}\right\|^{1 / 2}}-l_{2} e_{1}\left\|e_{1}\right\|^{p-1}+e_{1}^{*} .
$$

Taking the derivative of $e_{1}^{*}$ gives:

$$
\begin{aligned}
\dot{e}_{1}^{*} & =\dot{z}_{2}-\dot{\Delta}_{1} \\
& =-l_{3} \frac{e_{1}}{\left\|e_{1}\right\|}-\dot{\Delta}_{1} .
\end{aligned}
$$

As a result, the error dynamics of the observer for $\Delta_{1}$ can be represented as:

$$
\left\{\begin{array}{l}
\dot{e}_{1}=-l_{1} \frac{e_{1}}{\left\|e_{1}\right\|^{1 / 2}}-l_{2} e_{1}\left\|e_{1}\right\|^{p-1}+e_{1}^{*} \\
\dot{e}_{1}^{*}=-l_{3} \frac{e_{1}}{\left\|e_{1}\right\|}-\dot{\Delta}_{1}
\end{array} .\right.
$$

On the basis of the result in [54], when the observer gains $l_{1}$, $l_{2}$, and $l_{3}$ satisfy the condition (23), $e_{1}$ and $e_{1}^{*}$ can uniformly converge to the origin within fixed time:

$$
\begin{aligned}
t_{o} \leq & \left(\frac{1}{l_{2}(p-1) \varepsilon^{p-1}}+\frac{2(\sqrt{2} \varepsilon)^{1 / 2}}{l_{1}}\right) \\
& \times\left(1+\frac{l_{3}+L}{\left(l_{3}-L\right)\left(1-\sqrt{2 l_{3}} / l_{1}\right)}\right),
\end{aligned}
$$

where $\varepsilon>0$. The minimum value of $t_{o}(\varepsilon)$ is obtained as long as $\varepsilon=\left(2^{1 / 4} l_{1} / l_{2}\right)^{\frac{1}{p+1 / 2}}$. Recalling the definition $e_{1}^{*}=z_{2}-\Delta_{1}$, it is proven that $z_{2}$ can approach to $\Delta_{1}$ within fixed time.

Moreover, the proof procedure of the fixed-time observation of $\Delta_{3}$ is akin to that of $\Delta_{1}$. For the sake of space, the details are omitted herein.

Remark 8: In most of the observer design approaches, the estimation error can vanish asymptotically or within finite time. In contrast, the developed observers are capable of estimating $\Delta_{1}$ and $\Delta_{3}$ within fixed time. According to Eqs. (21)-(22), there are three observer gains for each observer. The purpose of selecting $l_{i}(i=1,2,3)$ is to ensure that the estimation errors $\left(\dot{e}_{1}\right.$ and $\left.\dot{e}_{1}^{*}\right)$ can converge to the origin within fixed time. To be more specific, the obtained condition $l_{3}>4 L_{1}$ drives $\dot{e}_{1}^{*}$ to zero within fixed time. Subsequently, the conditions, $l_{1}>\sqrt{2 l_{3}}$ and $l_{2}>0$, enable $\dot{e}_{1}$ to reach to the origin within fixed time. Hence, this property is important especially for safety-critical aerospace engineering systems.

\section{Finite-Time Fault-Tolerant Control Design}

With respect to Eq. (18), let $\tilde{y}_{1}=y_{1}$ and $\tilde{y}_{2}=y_{2}+\hat{\Delta}_{1}$, where $\hat{\Delta}_{1}$ denotes the estimated value of $\Delta_{1}$ that can be achieved by the proposed fixed-time observer. Define an integral terminal sliding manifold as:

$$
S=\tilde{y}_{2}+\int_{0}^{\tau} k_{1}\left\|\tilde{y}_{1}\right\|^{r_{1}} \frac{\tilde{y}_{1}}{\left\|\tilde{y}_{1}\right\|}+k_{2}\left\|\tilde{y}_{2}\right\|^{r_{2}} \frac{\tilde{y}_{2}}{\left\|\tilde{y}_{2}\right\|} d \tau,
$$

where $k_{1}, k_{2}>0, r_{2} \in(0,1)$, and $r_{1}=2 r_{2} /\left(2-r_{2}\right)$. The aim is to drive $y_{1}$ to the origin along $S=0$ in finite time, despite that actuator faults and model uncertainties are present in the HGV.

Theorem 2: The HGV FTC law is formulated as:

$$
\begin{gathered}
u=- \\
-b^{-1}\left(a+k_{1}\left\|\tilde{y}_{1}\right\|^{r_{1}} \frac{\tilde{y}_{1}}{\left\|\tilde{y}_{1}\right\|}+k_{2}\left\|\tilde{y}_{2}\right\|^{r_{2}} \frac{\tilde{y}_{2}}{\left\|\tilde{y}_{2}\right\|},\right. \\
\left.+z_{4}+\dot{z}_{2}+\eta_{1} S+\eta_{2}\|S\|^{r_{3}} \frac{S}{\|S\|}\right)
\end{gathered}
$$

where $r_{3} \in(0,1)$. Thus, the proposed FTC law ensures that $y_{1}$ is steered to the origin along $S=0$ in finite time, when actuator faults and model uncertainties are present.

Proof. Given the FTC law (30), differentiating the sliding surface (29) along the faulty system (18) can render:

$$
\begin{aligned}
\dot{S} & =\dot{\tilde{y}}_{2}+k_{1}\left\|\tilde{y}_{1}\right\|^{r_{1}} \frac{\tilde{y}_{1}}{\left\|\tilde{y}_{1}\right\|}+k_{2}\left\|\tilde{y}_{2}\right\|^{r_{2}} \frac{\tilde{y}_{2}}{\left\|\tilde{y}_{2}\right\|} \\
& =\dot{y}_{2}+\dot{z}_{2}+k_{1}\left\|\tilde{y}_{1}\right\|^{r_{1}} \frac{\tilde{y}_{1}}{\left\|\tilde{y}_{1}\right\|}+k_{2}\left\|\tilde{y}_{2}\right\|^{r_{2}} \frac{\tilde{y}_{2}}{\left\|\tilde{y}_{2}\right\|} \\
& =a+b u+z_{4}+\dot{z}_{2}+k_{1}\left\|\tilde{y}_{1}\right\|^{r_{1}} \frac{\tilde{y}_{1}}{\left\|\tilde{y}_{1}\right\|}+k_{2}\left\|\tilde{y}_{2}\right\|^{r_{2}} \frac{\tilde{y}_{2}}{\left\|\tilde{y}_{2}\right\|} \\
& =\left(z_{4}-\hat{z}_{4}\right)-\eta_{1} S-\eta_{2}\|S\|^{r_{3}} \frac{S}{\|S\|}
\end{aligned}
$$

By letting $e_{z_{4}}=\hat{z}_{4}-z_{4}$ and $e_{z_{2}}=\hat{z}_{2}-z_{2}$, Eq. (31) can be simplified as:

$$
\dot{S}=-e_{z_{4}}-\eta_{1} S-\eta_{2}\|S\|^{r_{3}} \frac{S}{\|S\|} .
$$

Define a finite-time bounded function [41]:

$$
V_{1}\left(S, \tilde{y}_{1}, \tilde{y}_{2}\right)=\frac{1}{2}\left(S^{T} S+\tilde{y}_{1}^{T} \tilde{y}_{1}+\tilde{y}_{2}^{T} \tilde{y}_{2}\right)
$$

Note that the parameter $r_{i}(i=1,2)$ satisfies the condition $0<r_{i}<1$, which implies that $\left\|\tilde{y}_{i}\right\|^{r_{i}}<1+\left\|\tilde{y}_{i}\right\|$. By taking 
derivative of $V_{1}$ along dynamics (18), one obtains:

$$
\begin{aligned}
\dot{V}_{1}= & S^{T} \dot{S}+\tilde{y}_{1}^{T} \dot{\tilde{y}}_{1}+\tilde{y}_{2}^{T} \dot{\tilde{y}}_{2} \\
= & S^{T} \dot{S}+\tilde{y}_{1}^{T}\left(\tilde{y}_{2}-e_{z_{2}}\right) \\
& +\tilde{y}_{2}^{T}\left(\dot{S}-k_{1}\left\|\tilde{y}_{1}\right\|^{r_{1}} \frac{\tilde{y}_{1}}{\left\|\tilde{y}_{1}\right\|}-k_{2}\left\|\tilde{y}_{2}\right\|^{r_{2}} \frac{\tilde{y}_{2}}{\left\|\tilde{y}_{2}\right\|}\right) \\
\leq & \left\|S^{T} e_{z_{4}}\right\|+\left\|\tilde{y}_{1}\right\|\left(\left\|\tilde{y}_{2}\right\|+\left\|e_{z_{2}}\right\|\right) \\
& +\left\|\tilde{y}_{2}\right\|\left(\left\|e_{z_{4}}\right\|+\eta_{1}\|S\|+\eta_{2}(1+\|S\|)\right) \\
& +\left\|\tilde{y}_{2}\right\|\left(k_{1}\left(1+\left\|\tilde{y}_{1}\right\|\right)+k_{2}\left(1+\left\|\tilde{y}_{2}\right\|\right)\right) \\
\leq & \frac{\left\|S^{T}\right\|^{2}+\left\|e_{z_{4}}\right\|^{2}}{2}+\frac{\left\|\tilde{y}_{1}\right\|^{2}+\left\|\tilde{y}_{2}\right\|^{2}}{2}+\frac{\left\|\tilde{y}_{1}\right\|^{2}+\left\|e_{z_{2}}\right\|^{2}}{2} \\
& +\frac{\left\|\tilde{y}_{2}\right\|^{2}+\left\|e_{z_{4}}\right\|^{2}}{2}+\frac{\eta_{1}+\eta_{2}}{2}\left(\left\|\tilde{y}_{2}\right\|^{2}+\|S\|^{2}\right) \\
& +\frac{\eta_{2}^{2}+\left\|\tilde{y}_{2}\right\|^{2}}{2}+\frac{\left(k_{1}+k_{2}\right)^{2}+\left\|\tilde{y}_{2}\right\|^{2}}{2} \\
& +\frac{k_{1}\left(\left\|\tilde{y}_{1}\right\|^{2}+\left\|\tilde{y}_{2}\right\|^{2}\right)}{2}+k_{2}\left\|\tilde{y}_{2}\right\|^{2} \\
= & \left(\frac{1}{2}+\frac{\eta_{1}+\eta_{2}}{2}\right)\left\|S^{T}\right\|^{2}+\left(1+\frac{k_{1}}{2}\right)\left\|\tilde{y}_{1}\right\|^{2} \\
& +\left(2+\frac{k_{1}}{2}+\frac{\eta_{1}+\eta_{2}}{2}+k_{2}\right)\left\|\tilde{y}_{2}\right\|^{2} \\
& +\left(\left\|e_{z_{4}}\right\|^{2}+\frac{1}{2}\left\|e_{z_{2}}\right\|^{2}+\frac{\eta_{2}^{2}}{2}+\frac{\left(k_{1}+k_{2}\right)^{2}}{2}\right) \\
\leq & K_{V_{1}} V_{1}+L_{V_{1}},
\end{aligned}
$$

where $K_{V_{1}}=4+k_{1}+\eta_{1}+\eta_{2}+2 k_{2}$, and $L_{V_{1}}=\max \left(\left\|e_{z_{4}}\right\|^{2}+\frac{1}{2}\left\|e_{z_{2}}\right\|^{2}+\frac{\eta_{2}^{2}}{2}+\frac{\left(k_{1}+k_{2}\right)^{2}}{2}\right)$, respectively. Theorem 1 guarantees that the estimation errors $e_{z_{2}}$ and $e_{z_{4}}$ converge to zero in fixed time, which implies that $e_{z_{2}}$ and $e_{z_{4}}$ are bounded. In addition, $L_{V_{1}}$ is bounded. Therefore, it can be concluded that $V_{1}$ and the state $\tilde{y}_{i}$ will not escape to infinity before the convergence of the observer error dynamics. Since $e_{z_{2}}$ and $e_{z_{4}}$ approach to zero in fixed time, Eq. (32) in turn becomes:

$$
\dot{S}=-\eta_{1} S-\eta_{2}\|S\|^{r_{3}} \frac{S}{\|S\|},
$$

which is finite-time stable. As long as the sliding surface is reached, the equivalent dynamics can be obtained using $\dot{S}=0$ :

$$
\dot{\tilde{y}}_{2}+k_{1}\left\|\tilde{y}_{1}\right\|^{r_{1}} \frac{\tilde{y}_{1}}{\left\|\tilde{y}_{1}\right\|}+k_{2}\left\|\tilde{y}_{2}\right\|^{r_{2}} \frac{\tilde{y}_{2}}{\left\|\tilde{y}_{2}\right\|}=0 .
$$

In consequence, it can be proved that the dynamics of (36) is finite-time stabilized, which can be represented as:

$$
\left\{\begin{array}{l}
\dot{\tilde{y}}_{1}=\tilde{y}_{2} \\
\dot{\tilde{y}}_{2}=-k_{1}\left\|\tilde{y}_{1}\right\|^{r_{1}} \frac{\tilde{y}_{1}}{\left\|\tilde{y}_{1}\right\|}-k_{2}\left\|\tilde{y}_{2}\right\|^{r_{2}} \frac{\tilde{y}_{2}}{\left\|\tilde{y}_{2}\right\|}
\end{array} .\right.
$$

Select another Lyapunov function as:

$$
V_{2}=k_{1} \frac{\left\|\tilde{y}_{1}\right\|^{r_{1}+1}}{r_{1}+1}+\frac{\left\|\tilde{y}_{2}\right\|^{2}}{2} .
$$

The time derivative of $V_{2}$ along the proceeding dynamics (37) can be written as:

$$
\begin{aligned}
\dot{V}_{2} & =k_{1}\left\|\tilde{y}_{1}\right\|^{r_{1}-1} \dot{\tilde{y}}_{1}^{T} \tilde{y}_{1}-\tilde{y}_{2}^{T}\left(k_{1} \frac{\tilde{y}_{1}}{\left\|\tilde{y}_{1}\right\|^{1-r_{1}}}+k_{2} \frac{\tilde{y}_{2}}{\left\|\tilde{y}_{2}\right\|^{1-r_{2}}}\right) \\
& =k_{1}\left\|\tilde{y}_{1}\right\|^{r_{1}-1} \dot{\tilde{y}}_{1}^{T} \tilde{y}_{1}-k_{1}\left\|\tilde{y}_{1}\right\|^{r^{-1}} \tilde{y}_{2}^{T} \tilde{y}_{1} \\
& =-k_{2}\left\|\tilde{y}_{2}\right\|^{r_{2}-1} \tilde{y}_{2}^{T} \tilde{y}_{2} \\
& =-k_{2}\left\|\tilde{y}_{2}\right\|^{r_{2}-1} \tilde{y}_{2}^{T} \tilde{y}_{2} \\
& =-k_{2}\left\|\tilde{y}_{2}\right\|^{r_{2}+1}
\end{aligned}
$$

By applying LaSalles invariance principle, the set $\left\{\left(\tilde{y}_{1}, \tilde{y}_{2}\right)\right.$ : $\left.\dot{V}\left(\tilde{y}_{1}, \tilde{y}_{2}\right)=0\right\}$ consists of $\tilde{y}_{2}=0$, while the only invariant set inside $\tilde{y}_{2}=0$ is the origin $\tilde{y}_{1}=\tilde{y}_{2}=0$. As a result, the asymptotic convergence of $\tilde{y}_{1}$ and $\tilde{y}_{2}$ is ensured.
Consider the vector field (37) and the dilation [28]:

$$
\begin{aligned}
& \left(\tilde{y}_{1,1}, \tilde{y}_{1,2}, \tilde{y}_{1,3}, \tilde{y}_{2,1}, \tilde{y}_{2,2}, \tilde{y}_{2,3}\right) \mapsto \\
& \left(\kappa \tilde{y}_{1,1}, \kappa \tilde{y}_{1,2}, \kappa \tilde{y}_{1,3}, \kappa^{\frac{1}{2-r_{2}}} \tilde{y}_{2,1}, \kappa^{\frac{1}{2-r_{2}}} \tilde{y}_{2,2}, \kappa^{\frac{1}{2-r_{2}}} \tilde{y}_{2,3}\right)
\end{aligned}
$$

where $\kappa>0$. Hence, the vector field (37) is homogeneous of the degree of $\frac{r_{2}-1}{2-r_{2}}<0$. Based on [28], the closed-loop system (36) is globally finite-time stable. In this case, $\tilde{y}_{1}$ and $\tilde{y}_{2}$ approach to zero in finite time.

Remark 9: The reaching phase time and the sliding phase time are finite on the basis of (35) and (37), respectively. Thus, the finite-time stability is successfully incorporated in the FTC against HGV actuator malfunctions and model uncertainties. On the other hand, the control cost from the actuators demanded by the fixed-time control is much larger than that under the finite-time control [55]. With respect to post-fault HGVs, inappropriate control costs may induce the actuator amplitude saturation and even second damage of healthy actuators in the course of actuator fault accommodation. From this fundamental aspect, the finite-time control concept is chosen instead of the fixed-time control at the FTC design stage.

Remark 10: Considering that the fault recovery time of an HGV is limited, both the reaching phase time and the sliding phase time are finite in terms of (34) and (36), respectively. This feature is integrated into the FTC design, allowing the states of the faulty $\mathrm{HGV}$ to return to the equilibrium within finite time.

Remark 11: Multivariable SMC design for a hypersonic vehicle is focused in [40]. However, the design is transformed into a decoupled one. Instead, the developed approach of this study has twofold benefits: 1) the problem associated with the decoupled design is avoided; and 2) the FTC is designed, with the assurance that the globally finite-time stability is achieved in spite of actuator faults. These improvements have the potential of enhancing the safety of operational HGVs.

Remark 12: References [40]-[42] present finite-time observers of nonlinear systems. Motivated by [54], fixed-time disturbance observers are proposed in this study, especially for multivariable situations. In contrast to [40]-[42], the convergence time of the designed disturbance observer is independent of initial conditions, while elegant solutions can be achieved by vector form design, which is more suitable for the HGV safety requirement. In addition to observer design aspect, this paper explicitly considers "mismatched uncertainty" which is used to handle the condition of actuator faults and model uncertainties, while this type of uncertainty is not prescribed in the recent work [42].

Remark 13: $\dot{z}_{2}$ can be obtained by two methods. One option is to obtain $\dot{z}_{2}$ (observer state) directly from the fixed-time observer. However, $\dot{z}_{2}$ is not a continuous signal. Instead, $\dot{z}_{2}$ can be estimated on-line by the robust exact fixed-time differentiator [56]. The differentiator can be implemented if the higher order derivatives of the input are bounded and the fixedtime escape does not exist. The differentiator transient can be driven adequately short by properly tuning the differentiator parameters. 


\section{Numerical Simulations}

\section{A. HGV Flight Conditions}

The initial flight conditions of the selected $\mathrm{HGV}$ are: $V(0)=3000 \mathrm{~m} / \mathrm{s}, H(0)=3000 \mathrm{~m}, \mu(0)=0^{\circ}, \alpha(0)=2^{\circ}$, $\beta(0)=0^{\circ}$, and $p(0)=q(0)=r(0)=0$. The geometric parameters are: $m=641.7 \mathrm{~kg}, \bar{b}=\bar{c}=0.8 \mathrm{~m}, S_{r}=0.5024 \mathrm{~m}^{2}$, $I_{x x}=65.12 \mathrm{~kg} \cdot \mathrm{m}^{2}, I_{y y}=247.26 \mathrm{~kg} \cdot \mathrm{m}^{2}$, and $I_{z z}=$ $247.26 \mathrm{~kg} \cdot \mathrm{m}^{2}$.

\section{B. Simulation Scenarios}

The FTC without fixed-time observer and the proposed FTC are both examined. The actuator faults, model uncertainties, and measurement noises are considered in the simulation.

1) Actuator time-varying faults:

$$
\begin{aligned}
& \lambda_{1}=\left\{\begin{array}{l}
1, \quad 0 \leq t<4 \\
0.7-\frac{t-4}{5} \times 0.3 \quad 4 \leq t<9 \\
0.4, t \geq 9 \\
0, \quad 0 \leq t<4 \\
5+\frac{t-4}{5} \times 4 \quad 4 \leq t<9 \\
9, \quad t \geq 9
\end{array}\right. \\
& \rho_{1}=\left\{\begin{array}{l}
1, \quad 0 \leq t<4 \\
0.7-\frac{t-4}{5} \times 0.2 \quad 4 \leq t<9 \\
0.5, t \geq 9 \\
0, \quad 0 \leq t<4 \\
-9+\frac{t-4}{5} \times 18 \quad 4 \leq t<9 \\
9, \quad t \geq 9
\end{array}\right. \\
& \lambda_{2}, \\
& \lambda_{3}=\left\{\begin{array}{l}
1, \quad 0 \leq t<4 \\
0.7-\frac{t-4}{5} \times 0.2 \quad 4 \leq t<9 \\
0.5, t \geq 9 \\
0, \quad 0 \leq t<4 \\
9-\frac{t-4}{5} \times 19 \\
-10, \quad t \geq 9
\end{array} \quad 4 \leq t<9\right.
\end{aligned}
$$

2) According to [57], the maximal degree of the mismatch in the aerodynamic moment coefficients $\left(C_{l}, C_{m}\right.$, and $C_{n}$ ) is chosen as $20 \%$. The roll, pitch, and yaw moments of inertia $\left(I_{x x}, I_{y y}\right.$, and $\left.I_{z z}\right)$ are perturbed by $20 \%$ of the nominal values. Moreover, the maximum dispersion of the selected HGV mass is $20 \%$ of the nominal value.

3) The actuator dynamics are chosen as $40 /(s+40)$ in the simulation studies. In addition, the white noise with a mean of 0 and covariance of 0.01 is injected into each measurement channel.

To quantitatively assess the attitude tracking performance, three indices corresponding to $\mu, \alpha$, and $\beta$ are defined as:

$$
\sigma_{i}=\sqrt{\frac{1}{t_{2}-t_{1}} \int_{t_{1}}^{t_{2}}\left|y_{1, i}\right|^{2} d \tau}, i=1,2,3
$$

where $\left[t_{1}, t_{2}\right]$ covers the time frame of the simulation run and $y_{1, i}$ is the $i$ th element of $y_{1}$. The defined metric is the scalar valued $L_{2}$ norm, as a measure of average tracking performance [58]. The norm measures the root-mean-squared "average" of the tracking error. A smaller $L_{2}$ norm indicates smaller tracking error and thus better tracking performance.

\section{Simulation Results}

The tracking performance and tracking errors of the HGV attitude are shown in Fig. 3 and Fig. 4, respectively. Both the selected FTC schemes can ensure the safety of the faulty HGV. As can be seen from Fig. 3(a) and Fig. 4(a), the tracking error of bank angle under the developed FTC scheme is significantly smaller than that of the FTC scheme without fixed-time observer. It is illustrated in Fig. 3(b) and Fig. 4(b) that the AOA tracking performance achieved by the designed FTC is better in comparison to the FTC without fixed-time observer. Fig. 3(c) and Fig. 4(c) show that the presented scheme outperforms the comparing FTC with respect to sideslip angle. Hence, from Fig. 3 and Fig. 4, the tracking errors remain remarkably smaller during the entire maneuver when the proposed FTC is used. In terms of the curves, the tracking performance by the FTC without fixed-time observer is inferior to that of the proposed FTC. The closed-loop behavior remains excellent in the case of the developed FTC, although the tracking errors exhibit a slightly worse transient behavior. As is visible in Fig. 5, the actuators governed by the proposed FTC can satisfactorily handle the time-varying faults.

The improved rate of $\sigma_{1}$ from the FTC without fixedtime observer to the designed FTC is $41.56 \%$ (from $0.77^{\circ}$ to $0.45^{\circ}$ ). The measure of $\sigma_{2}$ is enhanced by $47.46 \%$ (from $0.59^{\circ}$ to $0.31^{\circ}$ ) when the selected FTC schemes are compared. With respect to $\sigma_{3}$, the studied FTC also attains the superior performance than that of the FTC without fixed-time observer, with $49.02 \%$ improvement of the defined metric (from $0.51^{\circ}$ to $0.26^{\circ}$ ). The performance indices emphasize that the FTC design approach is applicable not only for ensuring the HGV safety, but also for preserving the sound tracking performance.

\section{CONCLUSIONS AND FUTURE WORKS}

A new development of integrating fixed-time observers and finite-time control into a fault-tolerant control scheme is presented for a hypersonic gliding vehicle, which can handle actuator faults and model variations. The benefits of the algorithms include: 1) the estimation errors can be driven to zero within fixed time; 2) the fault-tolerant control law is constructed using the composite-loop design concept, by which the finite-time stability of the faulty closed-loop system can be ensured; and 3) multivariable situation is incorporated at the design stages of both observer and fault-tolerant control for avoiding the decoupled issues induced by formulating a multi-input and multi-output system as $m$ single-input systems. These improvements offer the potential to enhance the safety of hypersonic vehicles. The case studies based on a full nonlinear model of the hypersonic gliding vehicle dynamics demonstrate that the proposed fault-tolerant control scheme can effectively deal with scenarios involving actuator faults and model variations.

Despite that the proposed strategy is capable of tolerating flight actuator faults, issues of actuator saturation and sensor fault diagnosis have not yet been considered in the design. Investigation of these factors, which may affect the performance of the fault-tolerant control system, is one of our future works. In addition, hardware-in-the-loop simulation 


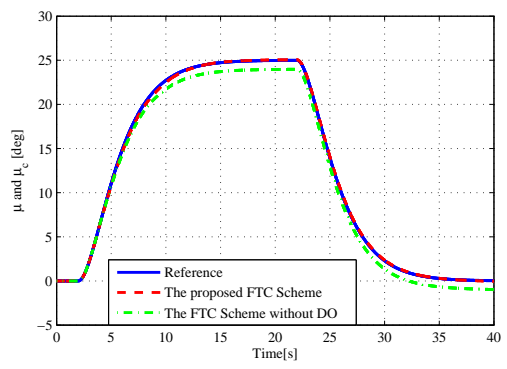

(a)

Fig. 3. The responses of $\mu, \alpha$, and $\beta$.

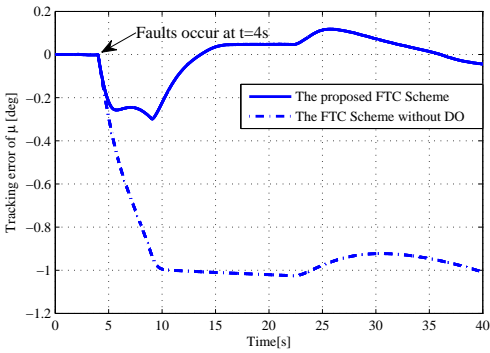

(a)

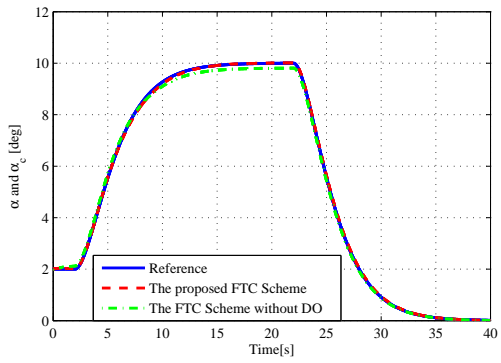

(b)

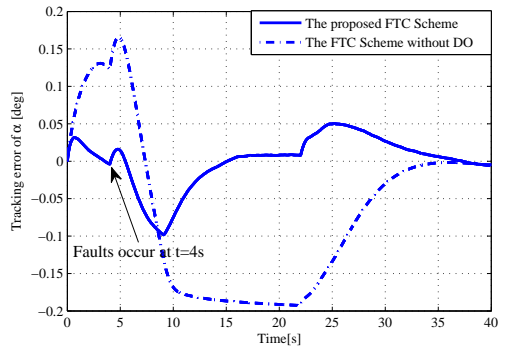

(b)

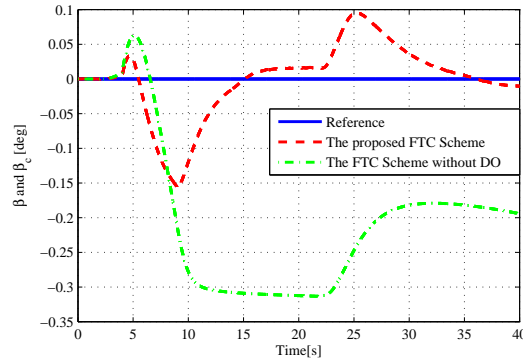

(c)

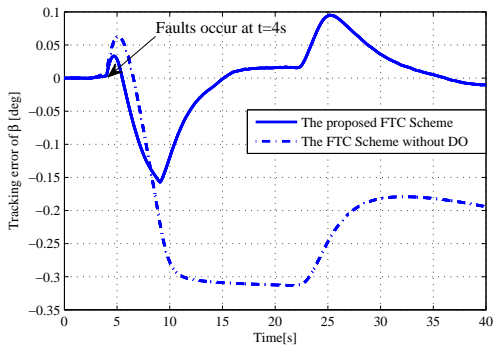

(c)

Fig. 4. The tracking errors of $\mu, \alpha$, and $\beta$.

experiment studies including designing testbed and validating the proposed algorithm should be conducted.

\section{ACKNOWLEDGEMENTS}

The authors would like to express their sincere gratitude to the Editor-in-Chief, the Associate Editor and the anonymous reviewers whose insightful comments have helped to improve the quality of this paper considerably.
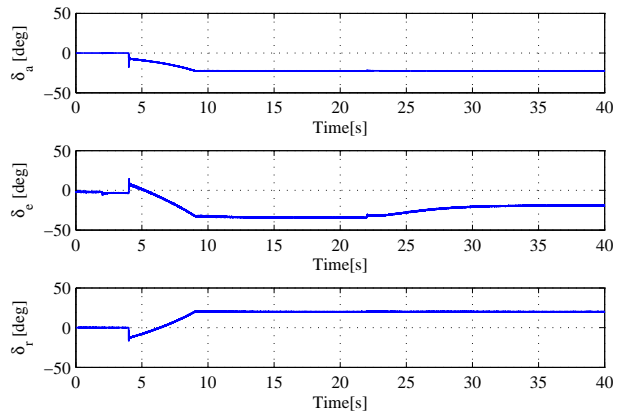

Fig. 5. The control signals $\delta_{a}, \delta_{e}$, and $\delta_{r}$.

\section{REFERENCES}

[1] C. C. Coleman and F. A. Faruqi, "On Stability and Control of Hypersonic Vehicles," Defence Science and Technology Organization, Edinburgh, Australia, Tech. Rep. DSTO-TR-2358, 2009.

[2] L. Fiorentini, A. Serrani, M. A. Bolender, and D. B. Doman, "Nonlinear Robust Adaptive Control of Flexible Air-Breathing Hypersonic Vehicles," J. Guid. Control Dynam., vol. 32, no. 2, pp. 401-416, 2009.
[3] D. Sigthorsson, P. Jankovsky, A. Serrani, S. Yurkovich, M. Bolender, and D. B. Doman, "Robust Linear Output Feedback Control of an Airbreathing Hypersonic Vehicle," J. Guid. Control Dynam., vol. 31, no. 4, pp. 1052-1066, 2008.

[4] L. Fiorentini and A. Serrani, "Adaptive Restricted Trajectory Tracking for a Non-Minimum Phase Hypersonic Vehicle Model," Automatica, vol. 48, no. 7, pp. 1248-1261, 2012.

[5] J. Yang, Z. H. Zhao, S. H. Li, and W. X. Zheng, "Composite Predictive Flight Control for Airbreathing Hypersonic Vehicles," Int. J. Control, vol. 87, no. 9, pp. 1970-1984, 2014.

[6] B. L. Tian, W. R. Fan, R. Su, and Q. Zong, "Real-Time Trajectory and Attitude Coordination Control for Reusable Launch Vehicle in Reentry Phase," IEEE Trans. Ind. Electron., vol. 62, no. 3, pp. 1639-1650, 2015.

[7] H. An, J. X. Liu, C. H. Wang, and L. G. Wu, "Disturbance ObserverBased Antiwindup Control for Air-Breathing Hypersonic Vehicles," IEEE Trans. Ind. Electron., vol. 63, no. 5, pp. 3038-3049, 2016.

[8] R. Isermann, Fault-Diagnosis Systems: An Introduction from Fault Detection to Fault Tolerance. 1st ed. Berlin, Heidelberg: SpringerVerlag, 2005.

[9] J. Chen and R. J. Patton, Robust Model-Based Fault Diagnosis for Dynamic Systems. Boston, MA, USA: Kluwer Academic Publishers, 1999.

[10] M. Blanke, C. W. Frei, F. Kraus, R. J. Patton, and M. Staroswiecki, "What is Fault-Tolerant Control?", in Proc. 4th IFAC SAFEPROCESS, Budapest, Hungary, June 2000, pp. 40-51.

[11] M. Blanke, M. Kinnaert, J. Lunze, J. Staroswiecki, and M. Schroder, $D i-$ agnosis and Fault-Tolerant Control. 1st ed. Berlin, Heidelberg: SpringerVerlag, 2003.

[12] J. Chen, R. J. Patton, and Z. Chen, "Active Fault-Tolerant Flight Control Systems Design Using the Linear Matrix Inequality Method," Trans. Inst. Measurement Control, vol. 21, no. 2/3, pp. 77-84, 1999.

[13] J. Chen and R. J. Patton, "Fault-Tolerant Control Systems Design Using the Linear Matrix Inequality Method," in Proc. 2001 Europ. Control Conf., Porto, Portugal, Sep. 2001, pp. 1993-1998.

[14] M. Blanke, R. Izadi-Zamanabadi, R. Bogh, and Z. P. Lunau, "FaultTolerant Control Systems - A Holistic View," Control Eng. Practice, vol. 5, no. 5, pp. 693-702, 1997.

[15] Y. M. Zhang and J. Jiang, "Bibliographical Review on Reconfigurable Fault-Tolerant Control Systems," Annu. Rev. Control, vol. 32, no. 2, pp. 229-252, 2008.

[16] X. Yu and J. Jiang, "A Survey of Fault-Tolerant Controllers Based on Safety-Related Issues," Аnпи. Rev. Control, vol. 39, no. 1, pp. 46-57, 
2015.

[17] J. Jiang and X. Yu, "Fault-Tolerant Control Systems: A Comparative Study Between Active and Passive Approaches," Annu. Rev. Control, vol. 36 , no. 1 , pp. $60-72,2012$.

[18] M. Mahmoud, J. Jiang, and Y. M. Zhang, Active Fault Tolerant Control Systems: Stochastic Analysis and Synthesis. Berlin, Heidelberg: Springer-Verlag, 2003.

[19] B. Jiang, Z. F. Gao, P. Shi, and Y. F. Xu, "Adaptive Fault-Tolerant Tracking Control of Near-Space Vehicle Using Takagi-Sugeno Fuzzy Models," IEEE Trans. Fuzzy Syst., vol. 18, no. 5, pp. 1000-1007, 2010.

[20] Q. K. Shen, B. Jiang, and V. Cocquempot, "Fault-Tolerant Control for TFuzzy Systems with Application to Near-Space Hypersonic Vehicle with Actuator Faults," IEEE Trans. Fuzzy Syst., vol. 20, no. 4, pp. 652-665, 2012.

[21] X. X. Hu, H. R. Karimi, L. G. Wu, and Y. Guo, "Model Predictive Control-Based Nonlinear Fault Tolerant Control for Air-Breathing Hypersonic Vehicles," IET Control Theory Appl., vol. 8, no. 13, pp. 11471153,2014

[22] Z. F. Gao, B. Jiang, P. Shi, M. S. Qian, and J. X. Lin, "Active Fault Tolerant Control Design for Reusable Launch Vehicle Using Adaptive Sliding Mode Technique," J. Frank. Inst., vol. 349, no. 4, pp. 1543-1560, 2012.

[23] F. Y. Chen, Z. Wang, G. Tao, and B. Jiang, "Robust Adaptive FaultTolerant Control for Hypersonic Flight Vehicles with Multiple Faults," ASCE J. Aerosp. Eng., vol. 28, no. 4, pp. 04014111, 2015.

[24] Y. H. Ji, H. L. Zhou, and Q. Zong, "Adaptive Active Fault-Tolerant Control of Generic Hypersonic Flight Vehicles," Proc. Inst. Mech. Eng. I J. Syst. Control Eng., vol. 229, no. 2, pp. 130-138, 2015.

[25] D. Z. Xu, B. Jiang, and P. Shi, "Robust NSV Fault-Tolerant Control System Design Against Actuator Faults and Control Surface Damage Under Actuator Dynamics," IEEE Trans. Ind. Electron., vol. 62, no. 9, pp. 5919-5928, 2015.

[26] H. An, J. X. Liu, C. H. Wang, and L. G. Wu, "Approximate BackStepping Fault-Tolerant Control of the Flexible Air-Breathing Hypersonic Vehicle," IEEE/ASME Trans. Mechatronics, vol. 21, no. 3, pp. 1680-1691, 2016

[27] S. P. Bhat and D. S. Bernstein, "Continuous Finite-Time Stabilization of the Translational and Rotational Double Integrators," IEEE Trans. Autom. Control, vol. 43, no. 5, pp. 678-682, 1998.

[28] S. P. Bhat, and D. S. Bernstein, "Finite-Time Stability of Continuous Autonomous Systems," SIAM J. Control Optim., vol. 38, no. 3, pp. 751$766,2000$.

[29] B. Xiao and S. Yin, "Velocity-Free Fault Tolerant and Unertainty Attenuation Control for a Class of Nonlinear Systems," IEEE Trans. Ind. Electron., vol. 63, no. 7, pp. 4400-4411, 2016.

[30] B. Xiao, S. Yin, and H. J. Gao, "Tracking Control of Robotic Manipulator with Uncertain Kinematics and Dynamics," IEEE Trans. Ind. Electron., vol. 63, no. 10, pp. 6439-6449, 2016.

[31] C. Edwards and Y. Shtessel, "Adaptive Dual Layer Super-Twisting Control and Observation," Int. J. Control, vol. 89, no. 9, pp. 1759-1766, 2016.

[32] X. R. Han, E. Fridman, S. K. Spurgeon, and C. Edwards, "On the Design of Sliding-Mode Static-Output-Feedback Controllers for Systems with State Delay," IEEE Trans. Ind. Electron., vol. 56, no. 9, pp. 3656-3664, 2009.

[33] C. Edwards and S. K. Spurgeon, Sliding Mode Control: Theory and Applications. Florida, USA: CRC Press, 1998.

[34] V. Andrieu, L. Praly, and A. Astolif, "Homogeneous Approximation, Recursive Observer and Output Feedback," SIAM J. Control Optim., vol. 47, no. 4, pp. 1814-1850, 2008.

[35] A. Polyakov, "Nonlinear Feedback Design for Fixed-Time Stabilization of Linear Control Systems," IEEE Trans. Autom. Control, vol. 57, no. 8, pp. 2106-2110, 2012

[36] A. Polyakov, D. Efimov, and W. Perruquetti, "Finite-Time and FixedTime Stabilization: Implicit Lyapunov Function Approach," Automatica, vol. 51, no. 1, pp. 332-340, 2015.

[37] M. Basin, C. B. Panathula, and Y. Shtessel, "Adaptive Uniform Finite/Fixed-Time Convergent Second-Order Sliding-Mode Control," Int. J. Control, vol. 89, no. 9, pp. 1777-1787, 2016.

[38] A. Polyakov, D. Efimov, and W. Perruquetti, "Robust Stabilization of MIMO Systems in Finite/Fixed Time," Int. J. Robust Nonlin. Control, vol. 26, no. 1, pp. 69-90, 2016.

[39] Y. Shtessel, J. Buffington, and S. Banda, "Multiple Timescale Flight Control Using Reconfigurable Sliding Modes," J. Guid. Control Dynam., vol. 22, no. 6, pp. 873-883, 1999.

[40] R. Su, Q. Zong, B. L. Tian, and M. You, "Comprehensive Design of Disturbance Observer and Non-Singular Terminal Sliding Mode Control for Resuable Launch Vehicles," IET Control Theory Appl., vol. 9, no. 12, pp. 1821-1830, 2015.
[41] J. Yang, S. H. Li, J. Y. Su, and X. H. Yu, "Continuous Nonsingular Terminal Sliding Mode Control for Systems with Mismatched Disturbances," Automatica, vol. 49, no. 7, pp. 2287-2291, 2013

[42] B. L. Tian, L. H. Liu, H. C. Lu, and Z. Y. Zuo, "Multivariable Finite Time Attitude Control for Quadrotor UAV," IEEE Trans. Ind. Electron., Online Published, DOI: 10.1109/TIE.2017.2739700, 2017.

[43] F. Y. Chen, W. Lei, K. Zhang, G. Tao, and B. Jiang, "A Novel Nonlinear Resilient Control for a Quadrotor UAV via Backstepping Control and Nonlinear Disturbance Observer," Nonlin. Dynam., vol. 85, no. 2, pp. 1281-1295, 2016.

[44] F. Y. Chen, R. Jiang, K. Zhang, B. Jiang, and G. Tao, "Robust Backstepping Sliding-Mode Control and Observer-Based Fault Estimation for a Quadrotor UAV," IEEE Trans. Ind. Electron., vol. 63, no. 8, pp. 50445056, 2016.

[45] F. Y. Chen, L. Cai, B. Jiang, and G. Tao, "Direct Self-Repairing Control for a Helicopter via Quantum Multi-Model and Disturbance Observer," Int. J. Syst. Sci., vol. 47, no. 3, pp. 533-543, 2016.

[46] F. Y. Chen, K. K. Zhang, B. Jiang, and C. Y. Wen, "Adaptive Sliding Mode Observer based Robust Fault Reconfiguration for a Helicopter with Actuator Fault," Asian J. Control, vol. 18, no. 4, pp. 1558-1565, 2016.

[47] K. W. Lee and S. N. Singh, "Robust Higher-Order Sliding-Mode FiniteTime Control of Aeroelastic Systems," J. Guid. Control Dynam., vol. 37, no. 5, pp. 1664-1670, 2014.

[48] J. L. Hunt, G. Laruelle, and A. Wagner, "Systems Challenges for Hypersonic Vehicles," NASA Langley Research Center, Hampton, Virginia, Tech. Rep. NASA-TM-112908, 1997.

[49] E. Baumann, J. W. Pahle, and M. C. Davis, "X-43A Flush Airdata Sensing System Flight-Test Results," J. Spacecraft Rockets, vol. 47, no. 1, pp. 48-61, 2000.

[50] X. Yu and J. Jiang, "Hybrid Fault-Tolerant Flight Control System Design Against Partial Actuator Failures," IEEE Trans. Control Syst. Technol., vol. 20, no. 4, pp. 871-886, 2012.

[51] H. Alwi, C. Edwards, and C. P. Tan, "Sliding Mode Estimation Schemes for Incipient Sensor Faults," Automatica, vol. 45, no. 7, pp. 1679-1685, 2009.

[52] X. G. Yan and C. Edwards, "Sensor Fault Detection and Isolation for Nonlinear Systems based on a Sliding Mode Observer," Int. J. Adapt. Control, vol. 21, no. 8-9, pp. 657-673, 2007.

[53] C. Edwards and X. G. Yan, "Sensor Fault Tolerant Control Using Sliding Mode Observers," Control Eng. Practice, vol. 14, no. 8, pp. 897-908, 2006.

[54] M. Basin, C. B. Panathula, and Y. Shtessel, "Multivariable Continuous Fixed-Time Second-Order Sliding Mode Control: Design and Convergence Time Estimation," IET Control Theory Appl., vol. 11, no. 8, pp. 1104-1111, 2017.

[55] B. L. Tian, Z. Y. Zuo, X. M. Yan, and H. Wang, "A Fixed-Time Output Feedback Control Scheme for Double Integrator Systems," Automatica, vol. 80, pp. 17-24, 2017.

[56] M. T. Angulo, J. A. Moreno, and L. Fridman, "Robust Exact Uniformly Convergent Arbitrary Order Differentiator," Automatica, vol. 49, no. 8, pp. 2489-2495, 2013.

[57] B. Fidan, M. Mirmirani, and P. A. Ioannou, "Flight Dynamics and Control of Air-Breathing Hypersonic Vehicles: Review and New Directions," in Proc. 12th AIAA Int. Space Planes Hyper. Syst. Technol., Norfolk, Virginia, Dec. 2003, AIAA 2003-7081

[58] B. Yao and M. Tomizuka, "Smooth Robust Adaptive Sliding Mode Control of Manipulators with Guaranteed Transient Performance," $J$. Dyn. Syst. Meas. Control, vol. 118, no. 4, pp. 764-775, 1996.

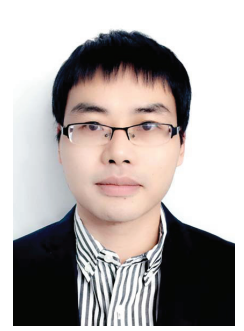

Xiang Yu (M'10-SM'13) received the B.S., M.S., and Ph.D. degrees in automatic control from Northwestern Polytechnical University, Xi'an, China, in 2003, 2004, and 2008, respectively. From 2009 to 2013, he was a postdoctoral research fellow at The University of Western Ontario, London, ON, Canada. He has been working at Concordia University since 2014. His main research interests include GNC for aerospace engineering systems, and fault-tolerant control. 


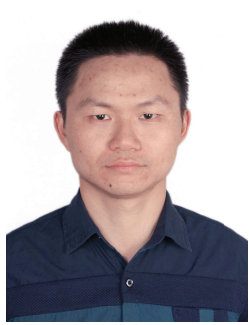

Peng Li received the B.S. degree in automation from Wuhan University, Wuhan, China, in 2004, and the M.S. and Ph.D. degrees in control science and engineering from National University of Defense Technology, Changsha, China, in 2006 and 2011, respectively. He is a Lecturer with the College of Mechatronics Engineering and Automation, National University of Defense Technology, and also with the State Key Laboratory of High Performance Computing in the same university. His current research interests include high order sliding-mode control and fault-tolerant flight control.

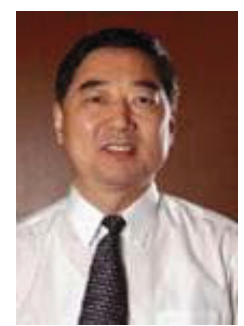

Youmin Zhang (M'99-SM'07) received the B.S., M.S., and Ph.D. degrees in automatic control from Northwestern Polytechnical University, Xi'an, China, in 1983, 1986, and 1995, respectively. Dr. Zhang is currently a Professor with the Department of Mechanical, Industrial \& Aerospace Engineering, Concordia University. His current research interests include fault diagnosis and fault-tolerant (flight) control systems, cooperative GNC of unmanned aerial/space/ground/surface vehicles. He has authored four books, over 450 journal and conference papers, and book chapters. Dr. Zhang is a Fellow of CSME, a Senior Member of AIAA and IEEE, Vice-President of International Society of Intelligent Unmanned Systems, and a member of the Technical Committee for several scientific societies. $\mathrm{He}$ is an Editorial Board Member, Editor-in-Chief, Editor-atLarge, Editor or Associate Editor of several international journals. He has served as the General Chair, the Program Chair, and IPC Member of several international conferences. 